\title{
Factors Associated with the Occurrence of Canine Parvoviral Enteritis in Dogs
}

\author{
V. Naveenkumar ${ }^{*}$, M. Vijaya Bharathi ${ }^{2}$, K. Porteen ${ }^{3}$, G. Selvaraju ${ }^{4}$ and K. Vijayarani $^{5}$ \\ ${ }^{1}$ Department of Veterinary Preventive Medicine, Madras Veterinary College, Tamil Nadu Veterinary and Animal Sciences \\ University (TANUVAS), Tamil Nadu, INDIA \\ ${ }^{2}$ Cattle and Buffalo Breeding unit, Post Graduate Research and Institute in Animal Sciences, Tamil Nadu Veterinary and \\ Animal Sciences University (TANUVAS), Tamil Nadu, INDIA \\ ${ }^{3}$ Department of Veterinary Public Health and Epidemiology, Madras Veterinary College, Tamil Nadu Veterinary and \\ Animal Sciences University (TANUVAS), Tamil Nadu, INDIA \\ ${ }^{4}$ Department of Veterinary Public Health and Epidemiology, Veterinary College and Research Institute, Namakkal, Tamil Nadu \\ Veterinary and Animal Sciences University (TANUVAS), Tamil Nadu, INDIA \\ ${ }^{5}$ Department of Animal Biotechnology, Madras Veterinary College, Tamil Nadu Veterinary and Animal Sciences University \\ (TANUVAS), Tamil Nadu, INDIA \\ "Corresponding author: VNaveenkumar; E-mail: naviviswanathan300@gmail.com
}
Received: 25 Sept., 2019
Revised: 16 Oct., 2019
Accepted: 20 Oct., 2019

\begin{abstract}
A prospective study was conducted to identify the risk factors associated with the incidence of Canine Parvoviral Enteritis (CPE) in dogs. Total of 120 animals screened using PCR assay, 72.50 percentage of animal were found positive for Canine Parvo Virus (CPV). Incidence in history of unvaccinated and vaccinated dogs was 79.69 and 64.29 per cent respectively. Age-wise predisposition of CPE indicated that the highest incidence was observed in both less than 3 months $(78.08 \%)$ and 3 to 6 months of age group (77.42\%) followed by 6 to 12 months of age group (34.50\%). Incidence of CPE in scheduled and unscheduled vaccination was 30.00 and 83.33 per cent respectively. In this study, unvaccinated status, unscheduled vaccination and young age groups are found to be significant risk factors associated with the occurrence of CPE.
\end{abstract}

Keywords: Incidence, CPE, unvaccinated dogs, unscheduled vaccination

Canine Parvo Virus (CPV) is an acute contagious viral disease of dog's especially young ones with high morbidity and mortality. It occurs as an intestinal form with haemorrhagic foul smelling diarrhoea and vomition with severe dehydration, while the cardiac form is seldom seen. The incidence of CPE is reported from various continents viz., Africa, Asia, Australia, America and Europe (Nandi and Kumar, 2010; Miranda and Thompson, 2016). Though the vaccination is the first step in controlling any viral diseases of dogs, CPV vaccine does not ensure $100 \%$ protection (Coyne, 2000). In general, HI titre of 1:80 is considered as a protective titre in puppies against CPV infection and vaccination will turn into immunization only when the titre level is below 1:10.

There are varieties of hindering factors contributing the occurrence of CPE in vaccinated animals which include MDA neutralization, lack of seroconversion variability in individuals, immune status of puppy, vaccination schedule, efficacy of vaccine, mutant virulence over the prototype vaccines and poor vaccine storage (Singh et al., 2013; Schultz, 2006; Nandi and Kumar, 2010). Though the various reasons are enlisted for CPE occurrence in vaccinated animals, neutralization of CPV by MDA and improper or irregular vaccination scheduled animals are the major reasons behind this outbreak (Singh et al., 2013). The associated risk factor analysis in CPE in vaccinated animals is seeking its demand to understand the disease

How to cite this article: Naveenkumar, V., Bharathi, M.V., Porteen, K., Selvaraju, G. and Vijayarani, K (2019). Factors associated with the occurrence of canine parvoviral enteritis in dogs. J. Anim. Res., 9(6): 893896. 
epidemic which may help to make an appropriate disease control strategy. This manuscript aimed to elucidate the role of vaccination and age as risk factors in the occurrence of CPE in vaccinated animals.

\section{MATERIALS AND METHODS}

In this prospective analysis, a total of 120 animals of varying breed and sex were included with the ailment of haemorrhagic foul-smelling diarrhoea and vomition with severe dehydration which was presented to Madras Veterinary College Teaching Hospital, Chennai, India. A structured questionnaire regarding age and vaccination history was collected to categorize the suspected animals into an age-wise category such as less than 3 months, $3-$ 6 months and 6 - 12 months of age group and vaccinated and unvaccinated groups. Among vaccinated animals scheduled vaccination to consist of one primary and 3 boosters at 21 days interval (WSAVA guidelines) and unscheduled vaccination consist of animals which do not follow the above protocol (Day et al., 2010).

From the suspected animals, stool samples were collected and processed for molecular confirmation. The DNA extraction from stool samples was performed by hot and boiling method (Schunck et al., 1995) and PCR was done with a published primer of Buonavoglia et al., 2001. The PCR was performed targeting $\mathrm{H}$ gene forward sequence (CAGGTGATGAATTTGCTACA) and reverse (CATTTGGATAAACTGGTGGT). The positive samples will yield a PCR product size of $630 \mathrm{bp}$ which is specific for CPV 2 confirmed cases (Fig. 1). In statistical analysis, Pearson chi square test and Odds ratio was calculated using SPSS package (Version 20.0).

\section{RESULTS AND DISCUSSION}

In this epidemiological study, $72.50 \% \mathrm{CPV}$ incidence was reported from the 120 suspected stool samples (Table 1). This is in agreement with Miranda et al. (2015) and Markovich et al. (2012), who has also documented $77.5 \%$ and $78 \%$ of CPV incidence by using PCR assay respectively. But, Mittal et al. (2015) and Godsall et al. (2010) documented the lower incidence of $6.74 \%$ and $58 \%$ by PCR respectively. The possible reason behind the higher incidence might be due to the framed animal inclusion criteria which are less than one year age group of animals with suspicion of haemorrhagic gastroenteritis
(Miranda et al., 2015). The lower incidence might be due to the inclusion of all similar systemic illness and which makes a decrease in the incidence pertaining to the study (Godsall et al., 2010).

Table 1: Age-wise incidence of Canine Parvoviral Enteritis

\begin{tabular}{cccc}
\hline Age group & $\begin{array}{c}\text { Total no. } \\
\text { of screened } \\
\text { animals }\end{array}$ & $\begin{array}{c}\text { Total no. } \\
\text { of positive } \\
\text { animals (\%) }\end{array}$ & $\chi^{\mathbf{2}-\text { value }}$ \\
\hline$<3$ months & 73 & $57(78.08)$ & $(11.348)^{* *}$, \\
$3-6$ months & 31 & $24(77.42)$ & $\mathrm{P}=0.003$ \\
$>6-12$ months & 16 & $6(34.50)$ & \\
\hline Total & $\mathbf{1 2 0}$ & $\mathbf{8 7}(\mathbf{7 2 . 5 0 )}$ & \\
\hline
\end{tabular}

On the age-wise incidence of CPV were analyzed and the higher positivity was encountered in both less than 3 months $(78.08 \%)$ and 3 to 6 months of age groups $(77.42 \%)$ and $34.50 \%$ of incidence in 6 to 12 months of age group (Table 1). The increased susceptibility of less than 6 months of age dogs for CPE were in agreement with other researchers (Umar et al., 2015; Thomas et al., 2014; Strepita et al., 2013) who documented the increased chance of CPV in less than 3 months followed by $3-6$ months and $6-12$ months of age group. Due to the affinity of CPV to the mitotic intestinal cells and gut flora and fauna changes especially during weaning time may facilitate the increased chances of CPV in less than 3 months of age group. Similarly, sudden fall in MDA level after 3 months of age makes 3 - 6 months age group as vulnerable for CPV infection in endemic areas (Strepita et al., 2013). On contradictory with the present study, Phukan et al., 2010 and Carmichael et al. (1983) documented the highest incidence of CPV with 7-12 months followed by $1-6$ months, and above 12 months age groups. The possible variation in these results might be due to improper vaccination schedule, lack of booster vaccination and improper storage of vaccines leads to vaccination failure in $7-12$ months as more prone to the disease (Carmichael et al., 1983).

Regarding vaccination status of dogs increased positivity noted in unvaccinated history dogs $(79.69 \%)$ than vaccinated $(64.29 \%)$ (Table 2$)$. The increased positivity of CPV in the unvaccinated population was documented many of the researchers (Houston et al., 1996; Parrish, 1999; Singh et al., 2013; Miranda and Thompson, 2016). In this study, more than 50 percent of CPV incidence was 
Table 2: Vaccination status-wise incidence of Canine Parvoviral Enteritis

\begin{tabular}{|c|c|c|c|c|c|c|}
\hline Category & $\begin{array}{c}\text { Total no. of } \\
\text { animal screened }\end{array}$ & $\begin{array}{c}\text { Total no. of } \\
\text { positive animals }\end{array}$ & $\begin{array}{l}\text { Percentage } \\
\text { positivity }\end{array}$ & $\begin{array}{l}\text { Odds } \\
\text { ratio }\end{array}$ & $\begin{array}{c}95 \% \text { Confidence } \\
\text { interval }\end{array}$ & P value \\
\hline \multicolumn{7}{|c|}{ Total no of animal screened $(n=120)$} \\
\hline Vaccinated animals & 56 & 36 & 64.29 & \multirow{2}{*}{2.179} & \multirow{2}{*}{$0.9620-4.940$} & $(3.554) \mathrm{NS}$ \\
\hline Unvaccinated animals & 64 & 51 & 79.69 & & & $\mathrm{P}=0.059$ \\
\hline \multicolumn{7}{|c|}{ Within the vaccinated animals $(n=56)$} \\
\hline Scheduled & 20 & 6 & 30.00 & \multirow{2}{*}{11.667} & \multirow{2}{*}{$3.188-42.692$} & $(15.929)^{* *}$ \\
\hline Unscheduled & 36 & 30 & 83.33 & & & $\mathrm{P}=0.000$ \\
\hline \multicolumn{7}{|c|}{ Within unscheduled animals $(n=36)$} \\
\hline Primary $(\mathrm{P})$ alone & 14 & 13 & 92.86 & 1 & - & - \\
\hline $\mathrm{P}+$ First booster $(\mathrm{B} 1)$ & 11 & 9 & 81.82 & 0.346 & $0.027-4.418$ & $\begin{array}{l}(0.711)^{\mathrm{NS}} \\
\mathrm{P}=0.399\end{array}$ \\
\hline $\mathrm{P}+\mathrm{B} 1+$ Second booster $(\mathrm{B} 2)$ & 11 & 8 & 72.73 & 0.593 & $0.078-4.498$ & $\begin{array}{l}(0.259)^{\mathrm{NS}} \\
\mathrm{P}=0.611\end{array}$ \\
\hline
\end{tabular}

observed in vaccinated animals and this might be due to improper or irregular vaccination, use of the poor quality vaccine and improper storage and/or handling of vaccine. The variation in the vaccination coverage might be due to adopting vaccination schedule based on endemic status, variation in incidence based on a location-wise, sampling frame of individual study and awareness among pet owners regarding the clean management and vaccination.

In this study increased incidence of CPV in unscheduled vaccination $(83.33 \%)$ was noted than scheduled vaccinated animals (30.00\%) (Table 2). The high incidence of CPV in unscheduled vaccinated animals was also reported by Castro et al., 2007 and Srinivas et al., 2013. Castro et al., 2007 documented about $93.47 \%$ incidence of CPV in unscheduled vaccinated animals.

Unscheduled vaccinated animals further identified as Primary, Primary + first booster and Primary + first booster + second booster and revealed 92.86, 81.82 and 72.73 percent respectively (Table 2). Among the unscheduled vaccinated animal category, higher incidence observed in primary followed by one and two booster injected animals. There are many hindering factors might responsible for the variation in the incomplete vaccinated animal group incidence viz., MDA neutralization, a sudden change in diet at weaning, immunity of individual dogs, nosocomial infections and co-infection. The above factors are almost in significant reasons despite MDA neutralization in individual animals play a major role in CPV occurrence (Parrish, 1999; Miranda and Thompson, 2016). Even after scheduled vaccination $30 \%$ of incidence was observed in our study. The possible reason behind this, $10 \%$ of puppies are not enough to get a protective titre due to the persisting maternal antibodies interference role (Miranda and Thompson, 2016).

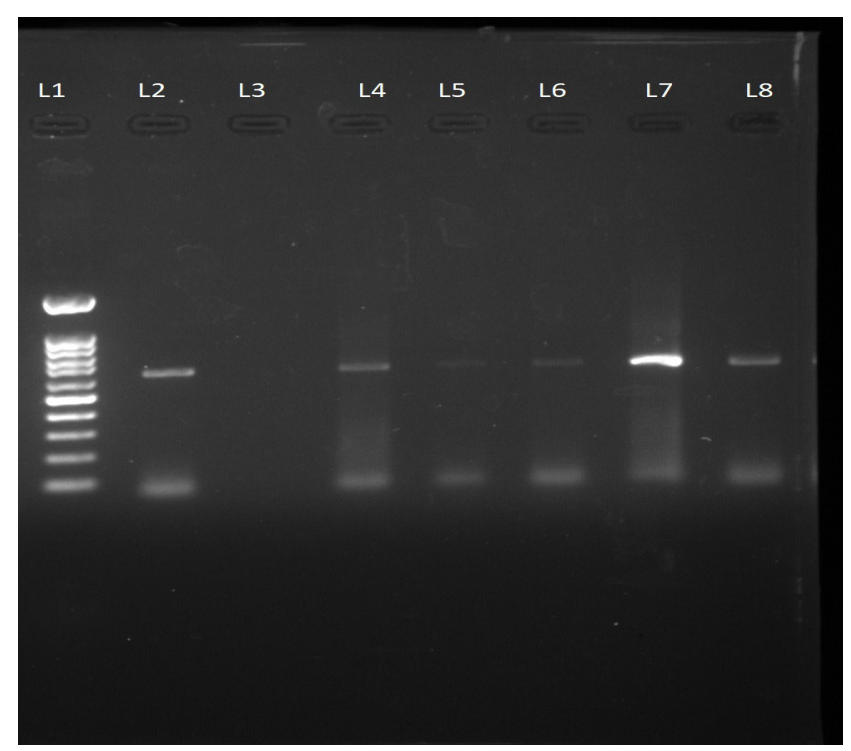

Fig. 1: Agarose gel (1.5\%) electrophoresis showing $\mathrm{H}$ primer PCR amplicon from CPV confirmed dog faeces. $\mathbf{L} 1-1.2 \mathrm{~kb}$ size ladder, L2 - Positive control, L3 - Negative control, L4- 8 - Positive samples for CPV 2 PCR product showing $630 \mathrm{bp}$ size

\section{CONCLUSION}

In this epidemiological study, out of 120 animals, $72.50 \%$ incidence of CPV was documented by using PCR assay. 
Unvaccinated animals, less than 6 months of age group and unscheduled vaccination are found to be significant risk factors associated with the occurrence of CPV. However using this descriptive study data, analytical oriented epidemiology studies are warranted to know the more risk factors associated with a disease outbreak in a different location.

\section{ACKNOWLEDGEMENTS}

This study is part of $\mathrm{Ph}$. D thesis submitted by the first author to Tamil Nadu Veterinary and Animal Sciences University (TANUVAS), India. This research was fully supported by TANUVAS. The authors were great thankful to Tamil Nadu Veterinary and Animal Sciences University (TANUVAS), India for supporting this epidemiological research in all aspects.

\section{REFERENCES}

Buonavoglia, C., Martella, V., Pratelli, A., Tempesta, M., Cavalli, A., Buonavoglia, D., Bozzo, G., Elia, G., Decaro, N. and Carmichael, L. 2001. Evidence for evolution of canine parvovirus type 2 in Italy. J. Gen. Virol., 82: 3021-3025.

Carmichael, L.E., Joubert, J.C. and Pollock, R.V. 1983. A modified live canine parvovirus vaccine. II. Immune response. Cornell Vet., 73: 13-29.

Castro, T.X., Miranda, S.C., Labarthe, N.V., Silva, L.E. and Cubel Garcia, R.C.N. 2007. Clinical and epidemiological aspects of canine parvovirus (CPV) enteritis in the State of Rio de Janeiro: 1995-2004. Arquivo brasileiro de medicina veterinária e zootecnia., 59(2): 333-339.

Coyne, M.J. 2000. Efficacy of Two Canine Parvovirus Vaccines for Inducing Seroconversion in Rottweiler and Doberman Pinscher Pups with Various Levels of Maternally Derived Antibodies. Vet. Therapeutics., 1(1): 35-42.

Day, M.J., Horzinek, M.C. and Schultz, R.D. 2010. WSAVA guidelines for the vaccination of dogs and cats. J. Small Anim. Pract., 51(6):e1-e32.

Godsall, S.A., Clegg, S.R., Stavisky, J.H., Radford, A.D. and Pinchbeck, G. 2010. Epidemiology of canine parvovirus and coronavirus in dogs presented with severe diarrhoea to PDSA PetAid hospitals. Vet. Rec., 167(6): 196-201.

Houston, D.M., Ribble, C.S. and Head, L.L. 1996. Risk factors associated with parvovirus enteritis in dogs: 283 cases (19821991). J. Am. Vet. Med. Assoc., 208(4): 542-546.

Markovich, J.E., Stucker, K.M., Carr, A.H., Harbison, C.E., Scarlett, J.M. and Parrish, C.R. 2012. Effects of canine parvovirus strain variations on diagnostic test results and clinical management of enteritis in dogs. J. Am. Vet. Med. Assoc., 241(1): 66-72.

Miranda, C. and Thompson, G. 2016. Canine Parvovirus: the worldwide occurrence of antigenic variants. J. Gen. Virol., 97: 2043-2057.

Miranda, C., Carvalheira, J., Parrish, C.R. and Thompson, G. 2015. Factors affecting the occurrence of canine parvovirus in dogs. Vet. Microbiol., 180(1-2): 59-64.

Mittal, M., Chakravarti, S., Mohapatra, J.K., Chug, P.K., Dubey, R., Upmanuyu, V., Narwal, P.S., Kumar, A., Churamani, C.P. and Kanwar, N.S. 2014. Molecular typing of canine parvovirus strains circulating from 2008 to 2012 in an organized kennel in India reveals the possibility of vaccination failure. Infect. Genet. Evol., 23:1-6.

Nandi, S. and Kumar, M. 2010. Canine parvovirus: current perspective. Indian J. Virol., 21(1): 31-44.

Parrish, C.R. 1999. Host range relationships and the evolution of canine parvovirus. Vet. Microbiol., 69: 29-40.

Phukan, A., Baishya, B., Deka, D. and Boro, P.K. 2010. Prevalence of canine parvovirus infection in Assam. Indian Vet. J., 87: 972-974.

Schultz R. 2006. Duration of immunity for canine and feline vaccines: a review. Vet. Microbiol., 117(1): 75-9.

Schunck, B., Kraft, W. and Truyen, U. 1995. A simple touchdown polymerase chain reaction for the detection of canine parvovirus and feline panleukopenia virus in feces. J. Virol. Method., 55: 427-33.

Singh, D., Verma, A.K., Kumar, A., Srivastava, M.K., Singh, S.K., Tripathi, A.K., Srivastava, A. and Ahmed, I. 2013. Detection of canine parvovirus by polymerase chain reaction assay and its prevalence in dogs in and around Mathura, Uttar Pradesh, India. Am. J. Biochem. Mol. Biol., 3(2): 264-270.

Srinivas, V. M.V., Mukhopadhyay, H.K., Thanislass, J., Antony, P.X. and Pillai, R.M. 2013. Molecular epidemiology of canine parvovirus in southern India, Vet. World., 6(10): 744-749.

Stepita, M.E., Bain, M.J. and Kass, P.H. 2013. Frequency of CPV infection in vaccinated puppies that attended puppy socialization classes. J. Am. Anim. Hosp. Assoc., 49: 95-100.

Thomas, J, Singh, M., Goswami, T.K., Verma, S. and Badasara, S.K. 2014. Polymerase chain reaction based epidemiological investigation of canine parvoviral disease in dogs at Bareilly region. Vet. World., 7(11): 929-932.

Umar, S., Ali, A., Younus, M., Maan, M.K., Shahzad, A., Khan, W.A. and Irfan, M. 2015. Prevalence of canine parvovirus infection at different pet clinics in Lahore, Pakistan. Pak. J. Zool., 47(3): 657-663. 